\title{
Observation of a topological crystalline insulator phase and topological phase transition in $\mathrm{Pb}_{1-x} \mathrm{Sn}_{x} \mathrm{Te}$
}

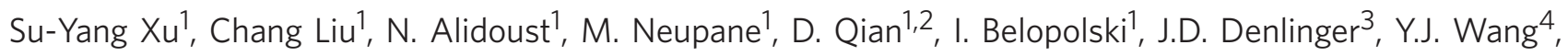

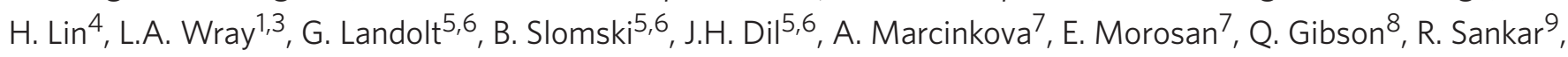
F.C. Chou 9 , R.J. Cava ${ }^{8}$, A. Bansil ${ }^{4} \&$ M.Z. Hasan ${ }^{1,10}$

A topological insulator protected by time-reversal symmetry is realized via spin-orbit interaction-driven band inversion. The topological phase in the $\mathrm{Bi}_{1-x} \mathrm{Sb}_{x}$ system is due to an odd number of band inversions. A related spin-orbit system, the $\mathrm{Pb}_{1-x} \mathrm{Sn}_{x} \mathrm{Te}$, has long been known to contain an even number of inversions based on band theory. Here we experimentally investigate the possibility of a mirror symmetry-protected topological crystalline insulator phase in the $\mathrm{Pb}_{1-x} \mathrm{Sn}_{x}$ Te class of materials that has been theoretically predicted to exist in its end compound $\mathrm{SnTe}$. Our experimental results show that at a finite $\mathrm{Pb}$ composition above the topological inversion phase transition, the surface exhibits even number of spinpolarized Dirac cone states revealing mirror-protected topological order distinct from that observed in $\mathrm{Bi}_{1}{ }_{-} \mathrm{Sb}_{x}$. Our observation of the spin-polarized Dirac surface states in the inverted $\mathrm{Pb}_{1-x} \mathrm{Sn}_{x} \mathrm{Te}$ and their absence in the non-inverted compounds related via a topological phase transition provide the experimental groundwork for opening the research on novel topological order in quantum devices.

\footnotetext{
${ }^{1}$ Joseph Henry Laboratory, Department of Physics, Princeton University, Princeton, New Jersey 08544, USA. ${ }^{2}$ Key Laboratory of Artificial Structures and Quantum Control (Ministry of Education), Department of Physics, Shanghai Jiao Tong University, Shanghai 200240, China. ${ }^{3}$ Advanced Light Source, Lawrence Berkeley National Laboratory, Berkeley, California 94305, USA. ${ }^{4}$ Department of Physics, Northeastern University, Boston, Massachusetts 02115, USA. ${ }^{5}$ Swiss Light Source, Paul Scherrer Institute, CH-5232, Villigen, Switzerland. ${ }^{6}$ Physik-Institute, Universitat Zurich-Irchel, CH-8057 Zurich, Switzerland. 7 Department of Physics and Astronomy, Rice University, Houston, Texas 77005, USA. ${ }^{8}$ Department of Chemistry, Princeton University, Princeton, New Jersey 08544, USA. ${ }^{9}$ Center for Condensed Matter Sciences, National Taiwan University, Taipei 10617, Taiwan. ${ }^{10}$ Princeton Center for Complex Materials, PRISM, Princeton University, Princeton, NJ 08544, USA. Correspondence and requests for materials should be addressed to M.Z.H. (email: mzhasan@princeton.edu).
} 
$\mathrm{T}$ opological insulators are band insulators are featuring uniquely spin-polarized surface states, which is believed to be a consequence of odd number of inversions of bulk bands driven by spin-orbit interaction alone or in combination with crystal lattice modulations ${ }^{1-5}$. The odd number of band inversion transitions in the bulk demarcate a $Z_{2}$ topological insulator (TI) phase with spin-polarized surface states from that of a conventional band insulator phase, as previously demonstrated in TI $\mathrm{Bi}_{1-x} \mathrm{Sb}_{x}$ and the $\mathrm{Bi}_{2} \mathrm{Se}_{3}$ series ${ }^{1-5}$. Within band theoretical calculations, lead tin telluride $\mathrm{Pb}_{1-x} \mathrm{Sn}_{x} \mathrm{Te}$, which is a narrow band gap semiconductor widely used for infrared optoelectronic and thermoelectric devices, ${ }^{6,7}$, has long been known to contain an even number of band inversions as electronic structure is tuned via the $\mathrm{Sn} / \mathrm{Pb}$ ratio ${ }^{8-13}$. Theoretical calculations have also predicted the occurrence of surface or interface states within the inverted band gap upon the band inversion transition ${ }^{11-13}$ but no experimental evidence of the surface states has been reported so far. Recent theoretical works $^{14,15}$ have further stimulated the experimental search for surface states in $\mathrm{Pb}_{1-x} \mathrm{Sn}_{x} \mathrm{Te}$. In a recent calculation ${ }^{15}$, Hsieh et al. predicted that the topological surface states in $\mathrm{SnTe}^{15}$ are protected by crystalline space group symmetries, in contrast to the time-reversal symmetry protection in the well-known $Z_{2}$ TIs.

The crystal group symmetry of $\mathrm{Pb}_{1-x} \mathrm{Sn}_{x} \mathrm{Te}$, critical for the realization of the topological crystalline insulator (TCI) phase ${ }^{14}$, is based on the sodium chloride structure (space group $\mathrm{Fm} \overline{3} \mathrm{~m}$ (225)). In this structure, each of the two atom types $(\mathrm{Pb} / \mathrm{Sn}$, or Te) forms a separate face-centred cubic lattice, with the two lattices interpenetrating so as to form a three-dimensional checkerboard pattern. The first Brillouin zone (BZ) of the crystal structure is a truncated octahedron with six square faces and eight hexagonal faces. The band gap of $\mathrm{Pb}_{1-x} \mathrm{Sn}_{x} \mathrm{Te}$ is found to be a direct gap located at the $L$ points in the $\mathrm{BZ}^{8}$, which are the centres of the eight hexagonal faces of the BZ. Owing to the inversion symmetry of the crystal, each $L$ point and its diametrically opposite partner on the $\mathrm{BZ}$ are completely equivalent. Thus, there are four distinct $L$ point momenta. It is well established that the band inversion transitions in the $\mathrm{Pb}_{1-} \mathrm{Sn}_{x} \mathrm{Te}$ take place at these four $L$ points of the $\mathrm{BZ}^{9-13}$. As a result, even number of (four) inversions exclude the system from being a Kane-Mele $\mathrm{Z}_{2} \mathrm{TI}^{2}$. However, it is interesting to note that the momentum space locations of the band inversions are coincident with the momentum space mirror plane within the BZ. This fact provides a clue that band inversions in $\mathrm{Pb}_{1-x} \mathrm{Sn}_{x} \mathrm{Te}$ may lead to a distinct topologically nontrivial phase that is irrelevant to the time-reversal symmetry but may be the consequence of the spatial mirror symmetries of the crystal, which has been theoretically shown in the inverted end compound $\mathrm{SnTe}^{15}$. However, it is experimentally known that SnTe crystals actually are subjected to a rhombohedral distortion ${ }^{16}$, which, strictly speaking, breaks the crystal mirror symmetries and hence excludes a gapless TCI phase ${ }^{15}$. More importantly, SnTe crystals are typically heavily $\mathrm{p}$-type $\mathrm{e}^{17}$ due to the fact that $\mathrm{Sn}$ vacancies are thermodynamically stable ${ }^{17}$, which makes the chemical potential of SnTe to lie below the bulk valence band maximum (VBM), consequently not cutting across the surface states. The surface states, which may exist within the band gap, are thus unoccupied and cannot be experimentally studied by photoemission experiments ${ }^{18}$. On the other hand, the $\mathrm{Pb}$-rich samples are reported to possess the ideal (averaged) sodium chloride structure without global rhombohedral distortion $^{16}$, which thus preserve the proper crystal symmetry required for the predicted TCI phase ${ }^{15}$. Moreover, it is possible to grow $\mathrm{Pb}$-rich crystals in which the chemical potential can be brought up above the bulk VBM (in-gap or even n-type) ${ }^{19}$, so as to lie near the surface state Dirac point.
Therefore, to experimentally investigate the possibility of a topological phase transition, and thus rigorously proving the existence of a TCI phase in the $\mathrm{Pb}_{1-x} \mathrm{Sn}_{x} \mathrm{Te}$ system, we hereby utilize angle-resolved photoemission spectroscopy (ARPES) and spin-resolved ARPES to study the low-energy electronic structure below and above the band inversion topological transition in the $\mathrm{Pb}$-rich compositional range of the system, through which the observation of spin-polarized surface states in the inverted regime and their absence in the non-inverted regime is demonstrated, correlating the observed spin-polarized Dirac surface states with the expected band inversion topological phase transition. We further map out the critically important spin structure of the surface states protected by mirror symmetries in the inverted topological composition. These results show that the topological order observed in $\mathrm{Pb}_{1-x} \mathrm{Sn}_{x} \mathrm{Te}$ is distinct from that previously discovered in $\mathrm{Bi}_{1-x} \mathrm{Sb}_{x}$ or $\mathrm{Bi}_{2} \mathrm{Se}_{3}$ (refs 1-5). Our spin-resolved study of $\mathrm{Pb}_{1-x} \mathrm{Sn}_{x}$ Te paves the way for further investigation of many novel properties of topological crystalline order ${ }^{14,20-22}$ in real materials and devices.

\section{Results}

Comparison of non-inverted and inverted compositions. To capture the electronic structure both below and above the band inversion transition (theoretically predicted to be around $x \simeq 1 / 3$ (refs 9,10), we choose two representative compositions, namely $x=0.2$ and $x=0.4$, for detailed systematic studies. Figure $1 \mathrm{c}$ shows the momentum-integrated core level photoemission spectra for both compositions, where intensity peaks corresponding to tellurium $4 d$, tin $4 d$ and lead $5 d$ orbitals are observed. The energy splitting of the $\mathrm{Pb}$ orbital is observed to be larger than that of the Sn orbital, which is consistent with the stronger spin-orbit coupling of the heavier $\mathrm{Pb}$ nuclei. In addition, the spectral intensity contribution of the $\mathrm{Sn}$ peaks in the $x=0.4$ sample (red) is found to be higher than that of in the $x=0.2$ sample (blue), which is also consistent with the larger $\mathrm{Sn}$ concentration in the $x=0.4$ samples. We perform systematic low-energy electronic structure studies on these two representative compositions. As the lowenergy physics of the system is dominated by the band inversion at $L$ points ( $L$ points projected onto $\bar{X}$ points on the (001) surface), we present ARPES measurements with the momentum space window centred at the $\bar{X}$ point, which is the midpoint of the surface BZ edge (see Fig. 1b). Figures 2a,b show the ARPES Fermi surface and dispersion mappings of the $\mathrm{Pb}_{0.8} \mathrm{Sn}_{0.2} \mathrm{Te}$ sample $(x=0.2)$. The system at $x=0.2$ is observed to be gapped: No band is observed to cross the Fermi level in the Fermi surface maps (Fig. 2a). The dispersion measurements (Fig. 2b) reveal a single hole-like band below the Fermi level. This single hole-like band is observed to show strong dependence with respect to the incident photon energy (see Fig. 2b, and also detailed in Supplementary Figs S1-S3 and Supplementary Methods in the Supplementary Information), which reflects its three-dimensionally dispersive bulk valence band origin. As a qualitative guide to the ARPES measurements on $x=0.2$, we present firstprinciples-based electronic structure calculation on the noninverted end compound PbTe (Fig. 2c). Our calculations confirm that $\mathrm{PbTe}$ is a conventional band insulator, whose electronic structure can be described as a single hole-like bulk valence band in the vicinity of each $\bar{X}$ point, which is consistent with our ARPES results on $\mathrm{Pb}$-rich $\mathrm{Pb}_{0.8} \mathrm{Sn}_{0.2}$ Te. The three-dimensional nature of the calculated bulk valence band is revealed by its $k_{\mathrm{z}}$ evolution in Fig. 2c, which is in qualitative agreement with the incident photon energy dependence of our ARPES measurements shown in Fig. 2 b.

Now we present comparative ARPES measurements under identical experimental conditions and setups on the $\mathrm{Pb}_{0.6} \mathrm{Sn}_{0.4} \mathrm{Te}$ 
a

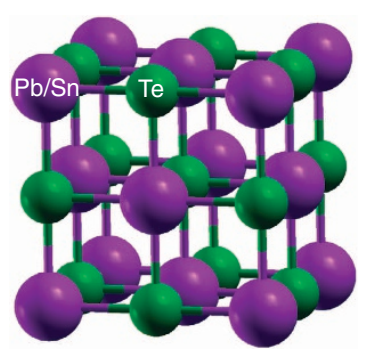

d

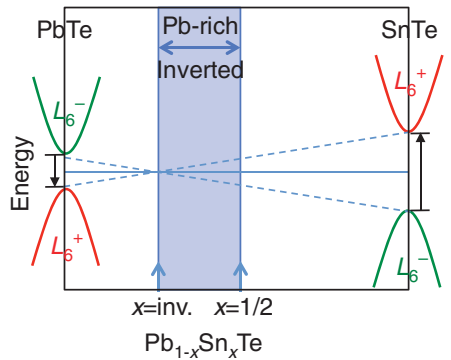

b

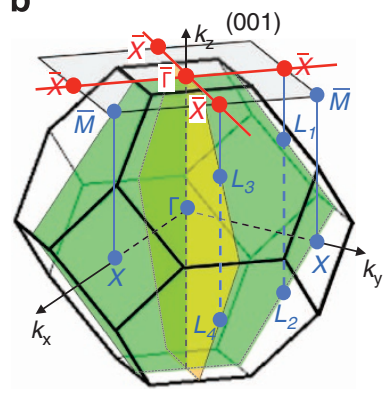

e

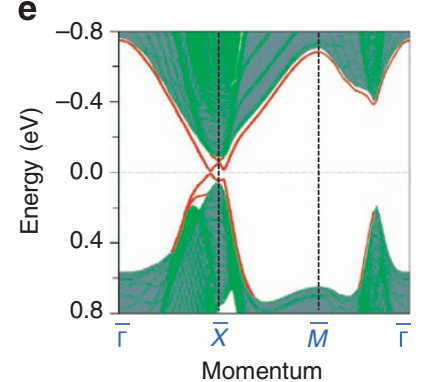

C

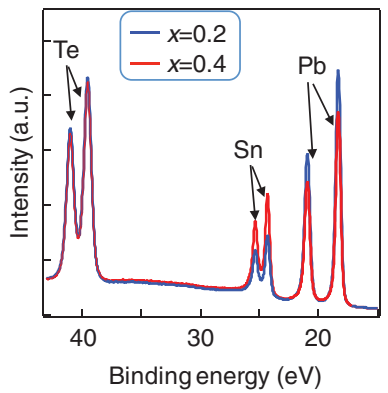

f

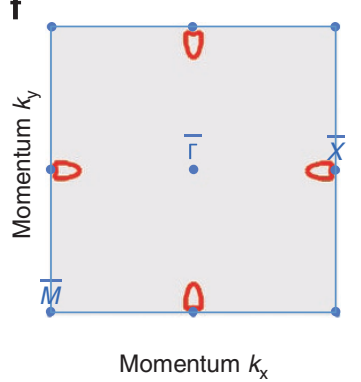

Figure 1 | Band inversion topological transition and surface states in $\mathbf{P b}_{\mathbf{1}_{-}} \mathbf{S n}_{\mathbf{x}} \mathbf{T e}$. (a) The lattice of $\mathrm{Pb}_{1-x} \mathrm{Sn}_{x} \mathrm{Te}$ system is based on the sodium chloride crystal structure. (b) The first $\mathrm{BZ}$ of $\mathrm{Pb}_{1-x} \mathrm{Sn}_{x} \mathrm{Te}$. The mirror planes are shown using green and light-brown colours. These mirror planes project onto the (001) crystal surface as the $\bar{X}-\bar{\Gamma}-\bar{X}$ mirror lines (shown by red solid lines). (c) ARPES measured core level spectra (incident photon energy 75 eV) of two representative compositions, namely $\mathrm{Pb}_{0.8} \mathrm{Sn}_{0.2} \mathrm{Te}$ and $\mathrm{Pb}_{0.6} \mathrm{Sn}_{0.4} \mathrm{Te}$. The photoemission core levels of tellurium $4 d$, tin $4 d$ and lead $5 d$ orbitals are observed. (d) The bulk band gap of $\mathrm{Pb}_{1-x} \mathrm{Sn}_{x} \mathrm{Te}$ alloy system undergoes a band inversion upon changing the $\mathrm{Pb} / \mathrm{Sn}$ ratio ${ }^{8-10}$. A TCl phase with metallic surface states is theoretically predicted when the band gap is inverted (towards $\mathrm{SnTe})^{15}$. The lowest-lying conduction and valence states associated with odd and even parity eigenvalues are labelled by $L_{6}^{-}$and $L_{6}^{+}$, respectively. First-principles-based calculation of band dispersion (e) and iso-energetic contour with energy set at $0.02 \mathrm{eV}$ below the Dirac node energy (f) of the inverted end compound SnTe as a qualitative reference for the ARPES experiments. The surface states are shown by the red lines whereas the bulk band projections are represented by the green shaded area in $\mathbf{e}$.

$(x=0.4)$ sample. In contrast to the conventional band insulator (insulating) behaviour in the $x=0.2$ sample, the Fermi surface mapping (Fig. $2 \mathrm{~d}$ ) on the $x=0.4$ sample shows two unconnected metallic Fermi pockets (dots) on the opposite sides of the $\bar{X}$ point. Such two-pockets Fermi surface topology cannot be easily interpreted as the bulk valence band as the low-energy bulk valence band of the $\mathrm{Pb}_{1-x} \mathrm{Sn}_{x}$ Te system is a single hole-like band, unless certain surface-umklapp processes of the bulk states are considered, which usually have only weak cross-section in ARPES measurements. The assignment of the observed two-Fermipockets as surface-umklapp processes or other bulk-related origins can be further ruled out by incident photon energy dependence $\left(k_{\mathrm{z}}\right.$ dispersion) measurements and our spin polarization studies of these metallic states (see Supplementary Figs S1-S5 in the Supplementary Information). The dispersion measurements on the $x=0.4$ sample are shown in Fig. 2e. The single hole-like bulk valence band, which is similar to that in the $x=0.2$ sample, is also observed below the Fermi level. More importantly, a pair of metallic states crossing the Fermi level on the opposite sides of the $\bar{X}$ point is observed along the $\bar{\Gamma}-\bar{X}-\bar{\Gamma}$ mirror line momentum space direction. These states are found to show no observable dispersion upon varying the incident photon energy (further details in Supplementary Fig. S3), reflecting its twodimensional character. On the other hand, the single hole-like band is observed to disperse strongly upon varying the incident photon energy, suggesting its three-dimensional character. At a set of different photon energy $\left(k_{\mathrm{z}}\right)$ values, the bulk valence band intensity overlaps (intermixing) with different parts of the surface states in energy and momentum space. At a photon energy of
$18 \mathrm{eV}$, the intermixing (intensity overlap) is strong, and the inner two branches of the surface states are masked by the bulk intensity. At photon energies of 10 and $24 \mathrm{eV}$, the surface states are found to be relatively better isolated. These ARPES measurements suggest that the $x=0.4$ sample lies on the inverted composition regime and that the observed surface states are related to the band inversion transition in $\mathrm{Pb}_{1-x} \mathrm{Sn}_{x} \mathrm{Te}$ as predicted theoretically ${ }^{11-13,15}$. As a qualitative guide, we present first-principles-based electronic structure calculation on the inverted end compound SnTe (Fig. 2f). The calculated electronic structure of SnTe is found to be a superposition of two $k_{\mathrm{z}}$ nondispersive metallic surface states and a single hole-like $k_{\mathrm{z}}$ dispersive bulk valence band in the vicinity of the $\bar{X}$ point, which is in qualitative agreement with the ARPES results on $\mathrm{Pb}_{0.6} \mathrm{Sn}_{0.4} \mathrm{Te}$.

Surface state topology of $\mathbf{P b}_{\mathbf{0 . 6}} \mathrm{Sn}_{\mathbf{0 . 4}} \mathrm{Te}$. We now perform systematic measurements on the surface electronic structure of the $\mathrm{Pb}_{0.6} \mathrm{Sn}_{0.4} \mathrm{Te}$. Figure $3 \mathrm{~b}$ shows the wide range Fermi surface mapping covering the first surface BZ. The surface states are observed to be present, and only present, along the mirror line $(\bar{\Gamma}-\bar{X}-\bar{\Gamma})$ directions. No other states are found along any other momentum directions on the Fermi level. In close vicinity to each $\bar{X}$ point, a pair of surface states are observed along the mirror line direction. One lies inside the first surface BZ whereas the other is located outside. Therefore, in total four surface states are observed within the first surface BZ, in agreement with the fact that there are four band inversions in $\mathrm{Pb}_{1-x} \mathrm{Sn}_{x}$ Te. The mapping zoomed- 
a

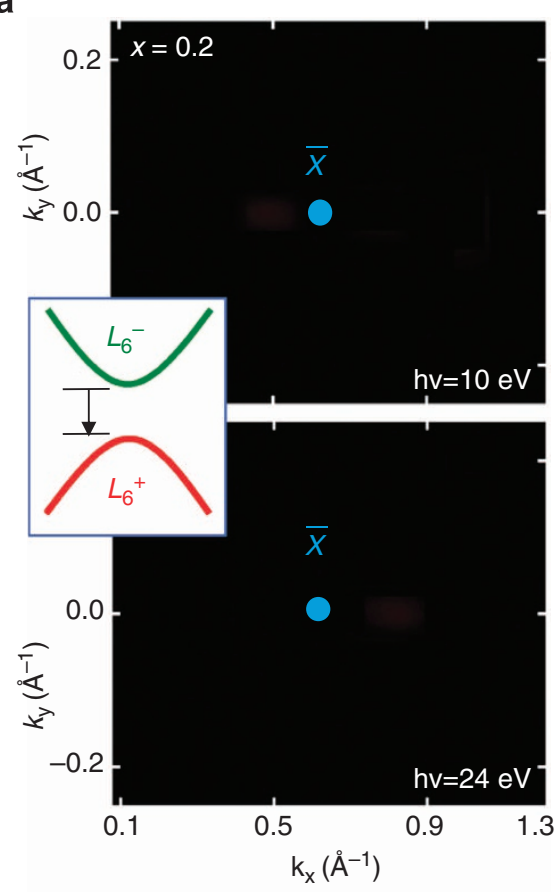

b

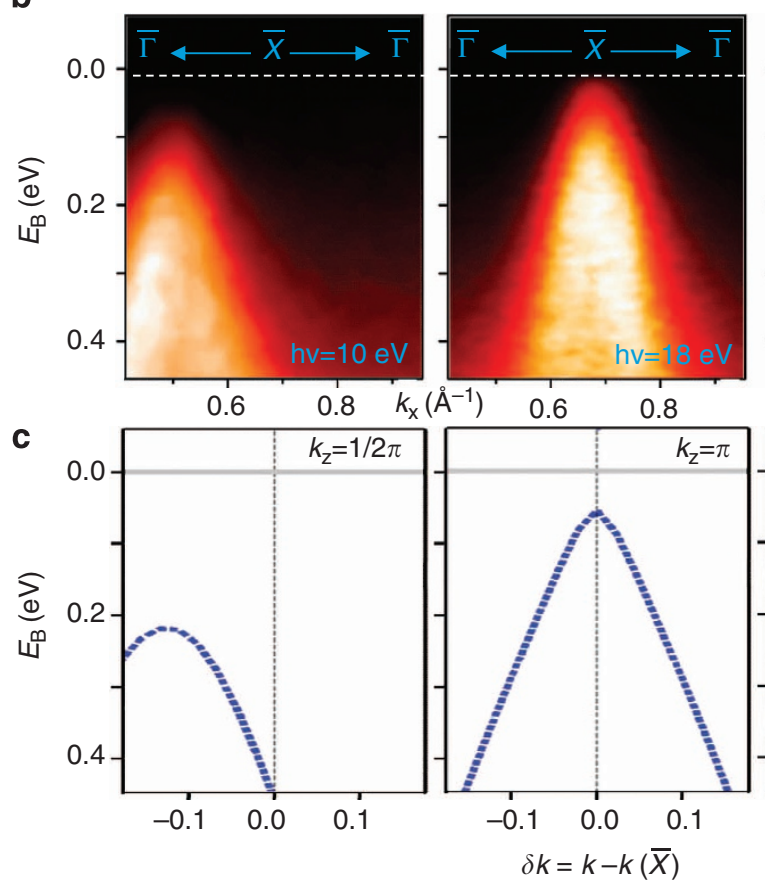

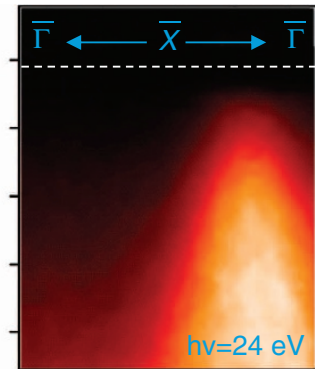

$0.6 \quad 0.8$

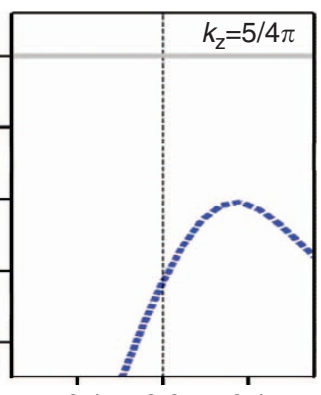

$\begin{array}{lll}-0.1 & 0.0 & 0.1\end{array}$

Low

d

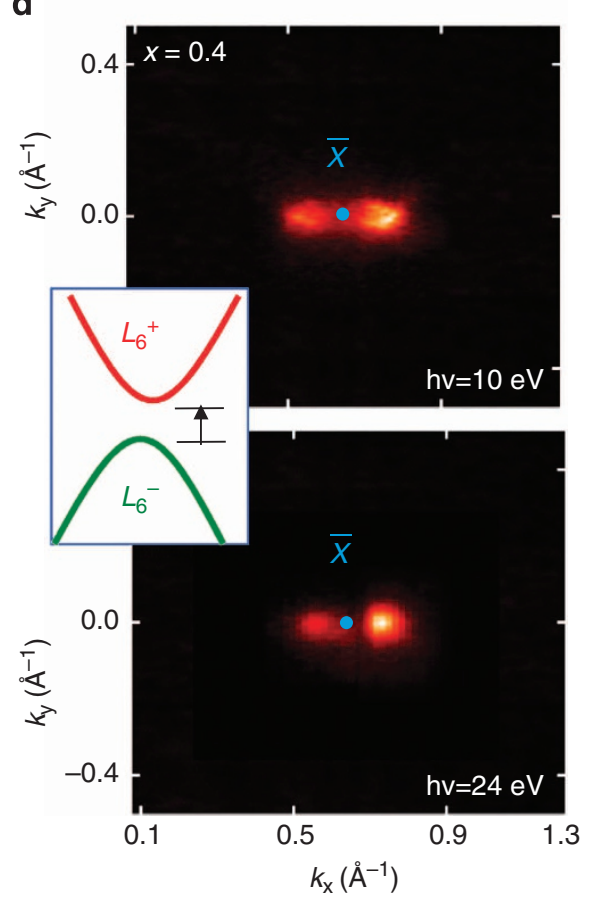

High

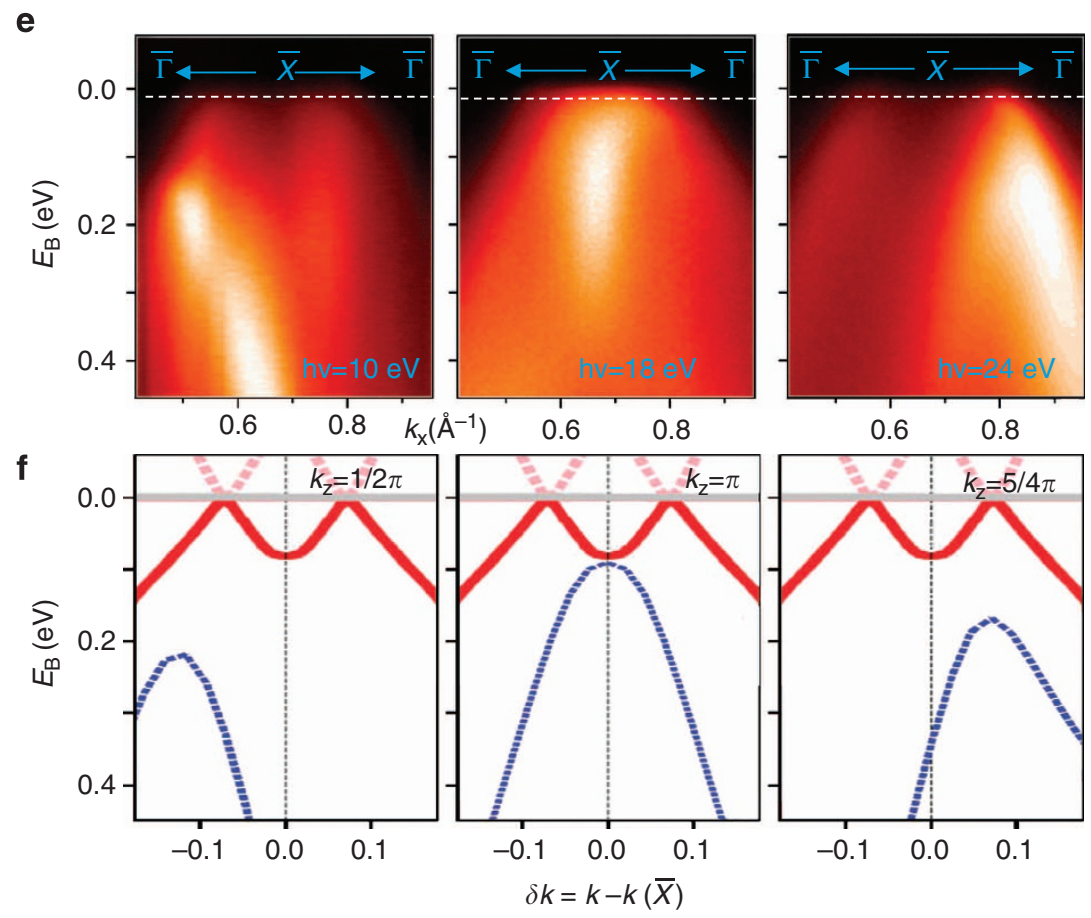

Figure 2 | Comparison of band insulator and TCI phases. (a,b) ARPES low-energy electronic structure measurements on $\mathrm{Pb}_{0.8} \mathrm{Sn}_{0.2} \mathrm{Te}(x=0.2)$. (c) Firstprinciples calculated electronic structure of $\mathrm{PbTe}(x=0)$ as a qualitative guide to the ARPES measurements on $\mathrm{Pb}_{0.8} \mathrm{Sn}_{0.2} \mathrm{Te}_{\mathrm{P}} \mathrm{PbTe}$, which lies on the noninverted regime, is theoretically expected to be a conventional band insulator ${ }^{15}$. (d,e) ARPES low-energy electronic structure measurements on $\mathrm{Pb}_{0.6} \mathrm{Sn}_{0.4} \mathrm{Te}_{\mathrm{e}}$ $(x=0.4)$ under identical experimental conditions and setups as in $\mathbf{a}$ and $\mathbf{b}$. (f) First-principles calculated electronic structure of $\operatorname{SnTe}(x=1)$ as a qualitative guide to the ARPES measurements on $\mathrm{Pb}_{0.6} \mathrm{Sn}_{0.4} \mathrm{Te}$. SnTe, which lies on the inverted regime, is theoretically expected to be a topological crystalline insulater $^{15}$. The insets of $\mathbf{a}$ and $\mathbf{d}$ show the non-inverted and inverted bulk band gap of the $x=0.2$ and $x=0.4$ samples, respectively. The blue and red lines in $\mathbf{c}$ and $\mathbf{f}$ are the calculated dispersion of the bulk bands and the surface states, respectively.

in near the $\bar{X}$ point (Fig. 3c) reveals two unconnected small pockets (dots). The momentum space distance from the centre of each pocket to the $\bar{X}$ point is about $0.09 \AA^{-1}$. Dispersion measurements $\left(E_{\mathrm{B}}\right.$ vs $\left.k\right)$ are performed along three important momentum space cuts, namely cuts 1,2 and 3 defined in Fig. $3 c$ to further reveal the electronic structure of the surface states. Metallic surface states crossing the Fermi level are observed in both cuts 1 and 2, whereas cut 3 is found to be fully gapped, 
a

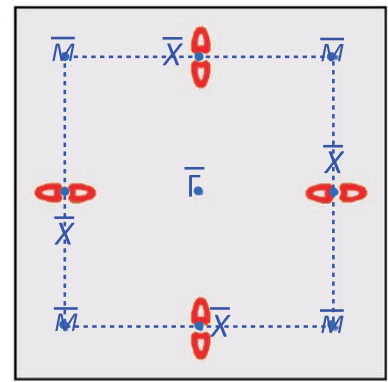

b

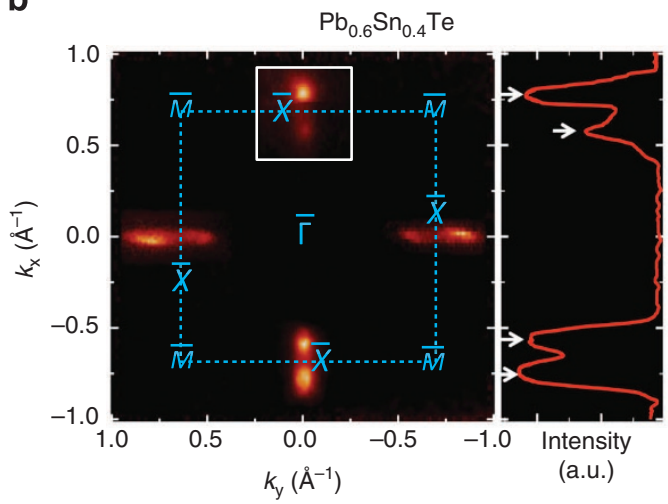

C

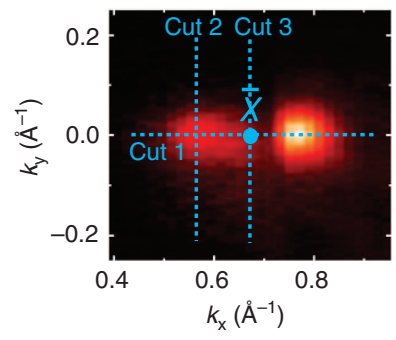

e

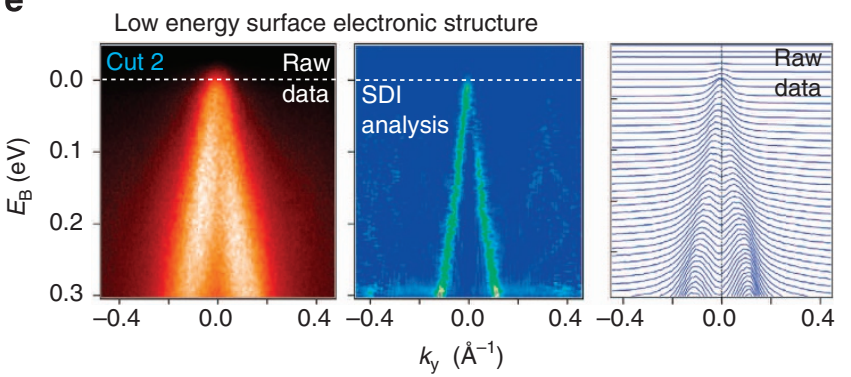

d

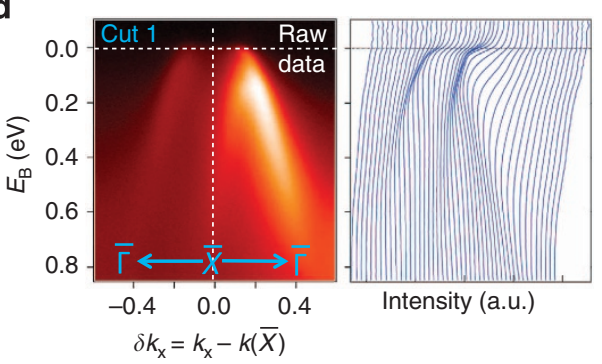

f

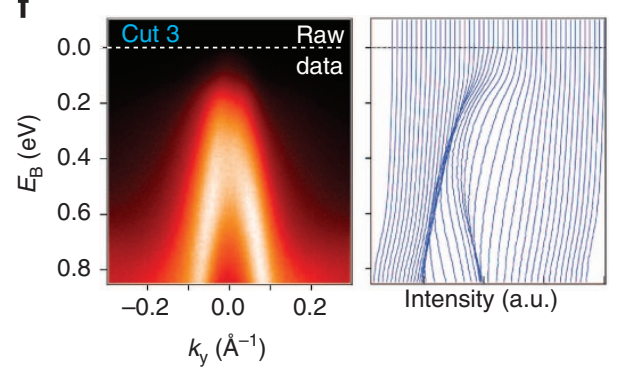

Figure 3 | Dirac surface states and $\mathbf{T C l}$ phase in $\mathbf{P b}_{\mathbf{0 . 6}} \mathbf{S n}_{\mathbf{0 . 4}} \mathbf{T e}$. (a) First-principles calculated surface states of SnTe with energy set at $0.02 \mathrm{eV}$ below the Dirac node energy are shown in red. The blue dotted lines mark the first surface BZ. (b) Left panel: ARPES iso-energetic contour mapping ( $E_{B}=0.02 \mathrm{eV}$ ) of $\mathrm{Pb}_{0.6} \mathrm{Sn}_{0.4}$ Te covering the first surface BZ using incident photon energy of $24 \mathrm{eV}$ (throughout Fig. 3). Right panel: spectral intensity distribution as a function of momentum along the horizontal mirror line (defined by $k_{y}=0$ ). The white arrows indicate the ARPES intensity peaks along this horizontal mirror line. (c) High-resolution Fermi surface mapping $\left(E_{\mathrm{B}}=0.0 \mathrm{eV}\right)$ in the vicinity of one of the $\bar{X}$ points (indicated by the white square in $\left.\mathbf{b}\right)$. (d-f) Dispersion maps $\left(E_{\mathrm{B}}\right.$ vs $k$ ) and corresponding energy (momentum) distribution curves of the momentum space, cuts 1, 2 and 3 . The momentum space cut-directions of cuts 1, 2 and 3 are defined by blue dotted lines in $\mathbf{c}$. The second derivative image (SDI) of the measured dispersion is additionally shown for $\mathbf{d}$.

which is consistent with the theoretically calculated surface states electronic structure shown in Fig. 1e. In cut 1 (Fig. 3d), which is the mirror line $(\bar{\Gamma}-\bar{X}-\bar{\Gamma})$ direction, a pair of surface states are observed on the Fermi level. The surface states in our $\mathrm{Pb}_{0.6} \mathrm{Sn}_{0.4} \mathrm{Te}$ samples are found to have a relatively broad spectrum, which can be possibly understood by the strong scattering in the disordered alloy system similar to the broad spectrum of the topological surface states in the $\mathrm{Bi}_{1-x} \mathrm{Sb}_{x}$ alloy ${ }^{23}$. In addition to the scattering broadening, the surface states are also observed to tail on the very strong main valence band emission (for example, the white region of intensity distribution in Fig. 3d). In the case of Fig. 2e (photon energy of $24 \mathrm{eV}$ ), the bulk VBM locates outside the first surface $\mathrm{BZ}$. The surface state inside the first surface BZ is relatively better isolated from the bulk bands as compared with the one outside the first surface BZ. We thus study the dispersion along cut 2 (Fig. 3d), which only cuts across the surface states inside the first surface BZ. Both the dispersion maps and the momentum distribution curves in cut 2 reveal that the surface states along cut 2 are nearly Dirac-like (linearly dispersive) close to the Fermi level. In many TIs, the surface states deviate from ideal linearity ${ }^{24}$. Fitting of the momentum distribution curves of cut 2 (see Supplementary Fig. S6 for data analysis) shows that the experimental chemical potential $\left(\mathrm{E}_{\mathrm{F}}\right)$ lies roughly at (or just below) the Dirac point energy $\left(\mathrm{E}_{\mathrm{D}}\right), \mathrm{E}_{\mathrm{F}}=\mathrm{E}_{\mathrm{D}} \pm 0.02 \mathrm{eV}$. The surface states' velocity is obtained to be $2.8 \pm 0.1 \mathrm{eV} \AA\left(4.2 \pm 0.2 \times 10^{5} \mathrm{~m} \mathrm{~s}^{-1}\right)$ along cut 2 , and $1.1 \pm 0.3 \mathrm{eV} \AA\left(1.7 \pm 0.4 \times 10^{5} \mathrm{~m} \mathrm{~s}^{-1}\right)$ for the two outer branches along cut 1 , respectively.

Spin polarization measurements of the surface states. We study the spin polarization of the low-energy states of the $\mathrm{Pb}_{0.6} \mathrm{Sn}_{0.4} \mathrm{Te}$ samples, which are highly dominated by the surface states near the Fermi level. We further compare and contrast their spin behaviour with that of the states at high binding energies away from the Fermi level, which are highly dominated by the bulk valence band in the same sample. Spin-resolved (SR) 
a

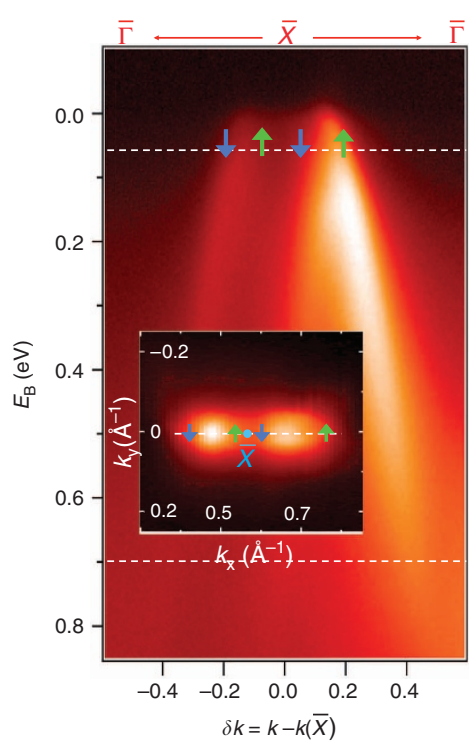

f

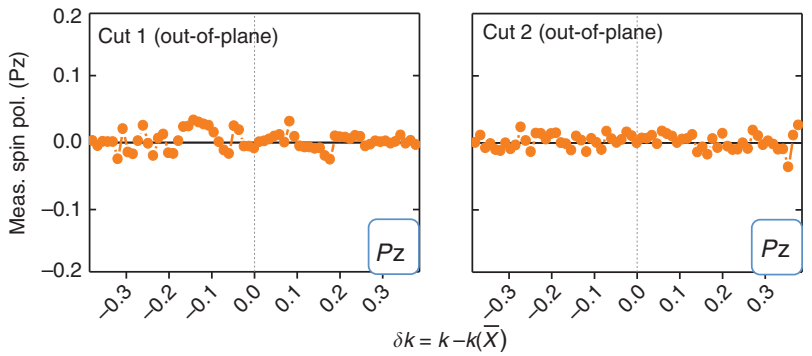

b

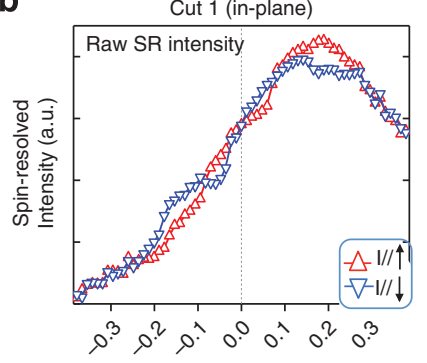

C

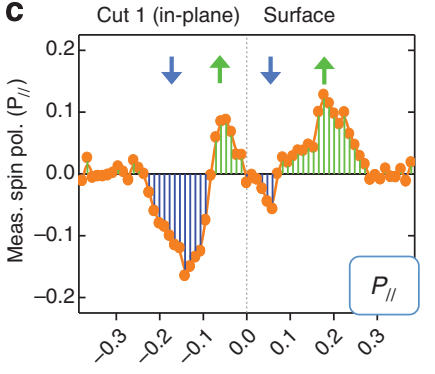

d $\quad$ Cut 2 (in-plane)

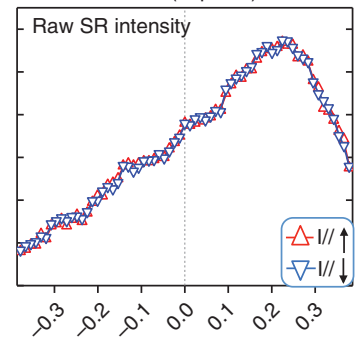

e Cut 2 (in-plane) Bulk

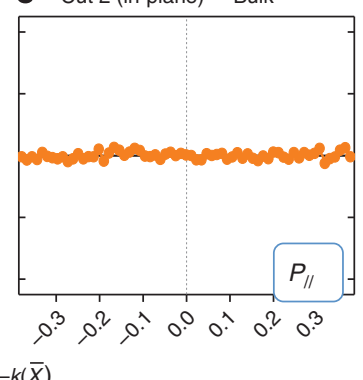

g

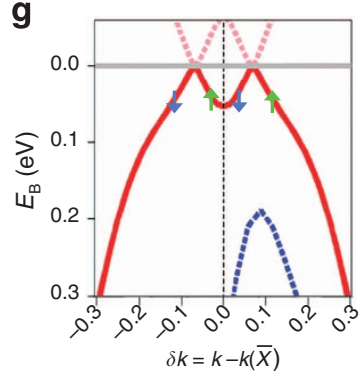

Figure 4 | Spin polarization of topological Dirac surface states. (a) ARPES dispersion map along the mirror line direction. The white dotted lines show the binding energies chosen for spin-resolved measurements, namely SR-Cut 1 at $E_{\mathrm{B}}=0.06 \mathrm{eV}$ and SR-Cut 2 at $E_{\mathrm{B}}=0.70 \mathrm{eV}$. Inset: Measured spin polarization profile is shown by the green and blue arrows on top of the ARPES iso-energetic contour at binding energy $E_{B}=0.06 \mathrm{eV}$ for SR-Cut1. Measured in-plane spin-resolved intensity (b) and in-plane spin polarization (c) of the surface states (SR-Cut 1) near the Fermi level at $E_{B}=0.06 \mathrm{eV}$. Measured in-plane spinresolved intensity $(\mathbf{d})$ and in-plane spin polarization (e) of the bulk valence bands (SR-Cut 2) at $E_{\mathrm{B}}=0.70 \mathrm{eV}$. (f) Out-of-plane spin polarization measurements of SR-Cut 1 and SR-Cut 2. The error bar is \pm 0.01 for data points in all spin polarization measurements. (g) Theoretically expected spin polarization configuration of the surface states which corresponds to a mirror topological invariant (the mirror Chern number) $n_{M}=-2($ refs 27,28 ). Measured spin polarization texture configuration (green and blue arrows) of the surface states (SR-Cut 1) is shown on top of the calculated surface states. The blue and red lines are the calculated bulk bands and surface states, respectively.

measurements are performed in the spin-resolved momentum distribution curve mode ${ }^{25,26}$, which measures the spin-resolved intensity and net spin polarization at a fixed binding energy along a certain momentum space cut direction (detailed in the Methods section). As shown in Fig. 4a, our spin-resolved measurements are performed along the mirror line $(\bar{\Gamma}-\bar{X}-\bar{\Gamma})$ direction, as the electronic and spin structure along this direction is most critically relevant to the predicted TCI phase ${ }^{15}$. Considering that the natural Fermi level of our $x=0.4$ samples are very close to the Dirac point (which is spin degenerate), the spin polarization of the surface states are measured at $60 \mathrm{meV}$ below the Fermi level to gain proper contrast, namely SR-Cut 1 in Fig. 4a. As shown in the net spin polarization measurement of SR-Cut 1 in Fig. 4c, in total four spins pointing in the $( \pm)$ in-plane tangential direction are revealed for the surface states along the mirror line direction. This is well consistent with the observed two surface state cones (four branches in total) near an $\bar{X}$ point along the mirror line direction. To compare and contrast the spin polarization behaviour of the surface states (SR-Cut 1) with that of the bulk states, we perform spin-resolved measurement SR-Cut 2 at $E_{\mathrm{B}}=0.70 \mathrm{eV}$, where the bulk valence bands are prominently dominated.
Indeed, in contrast to SR-Cut 1 reflecting the surface states' spin polarization, no significant net spin polarization is observed for SR-Cut2, which is expected for the bulk valence bands of the inversion symmetric (centrosymmetric) $\mathrm{Pb}_{1-x} \mathrm{Sn}_{x}$ Te system. Our observed spin polarization configuration of the surface states is also in qualitative agreement with the first-principles calculation spin texture of the SnTe TCI surface states (see Supplementary Figs S7-S9 for texture calculation); and experimental derived topological invariant (Mirror Chern number ${ }^{27,28}$ ) $n_{\mathrm{M}}=-2$ also agrees with theoretical prediction for $\mathrm{SnTe}^{15}$.

\section{Discussion}

We discuss the possibility that the observed surface states in our data are the signature of the theoretically predicted TCI phase. The TCI phase was predicted to be observed in the band inverted side and absent in the non-inverted side ${ }^{15}$. In our data of the $\mathrm{Pb}_{0.6} \mathrm{Sn}_{0.4} \mathrm{Te}$ samples, which lie on the inverted side, surface states are observed, and only observed, along the two independent mirror line $(\bar{\Gamma}-\bar{X}-\bar{\Gamma})$ directions. Within the first surface BZ, two surface states are observed on each mirror line, which are 
a
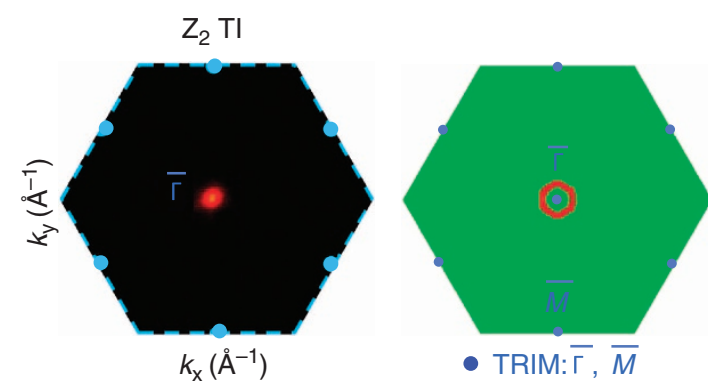

b

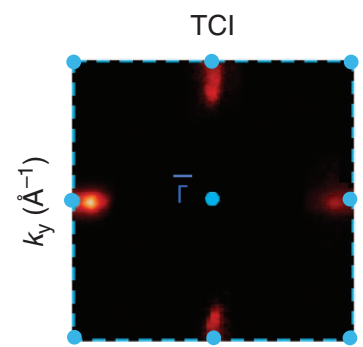

$k_{\mathrm{x}}\left(\AA^{-1}\right)$

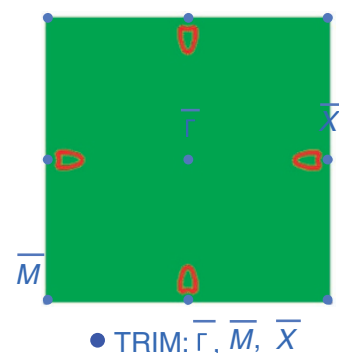

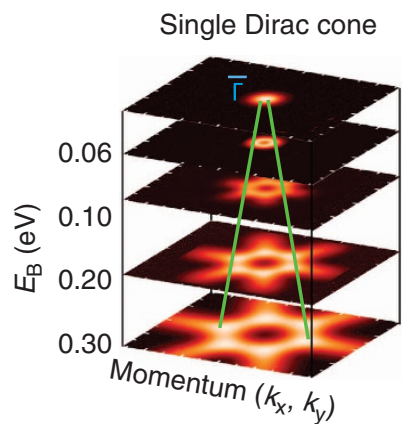

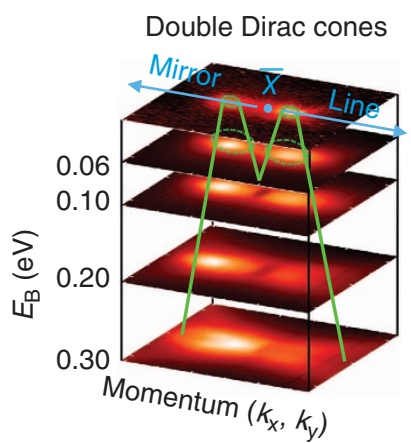

Figure 5 | A comparison of $\mathbf{Z}_{\mathbf{2}}$ (Kane-Mele) $\mathbf{T I}$ and $\mathbf{T C I}$ phases. (a) ARPES and calculation results of the surface states of a $\mathrm{Z}_{2} \mathrm{TI}_{\mathrm{GeBi}} \mathrm{Te} \mathrm{A}_{4}$ an analogue to $\mathrm{Bi}_{2} \mathrm{Se}_{3}{ }^{4}$. Left: ARPES measured Fermi surface with the chemical potential tuned near the surface Dirac point. Middle: first-principles calculated iso-energetic contour of the surface states near the Dirac point. Right: a stack of ARPES iso-energetic contours near the $\bar{\Gamma}$ point of the surface BZ. (b) ARPES measurements on the $\mathrm{Pb}_{0.6} \mathrm{Sn}_{0.4} \mathrm{Te}(x=0.4)$ samples and band calculation results on the end compound $\mathrm{SnTe}$. The green straight lines in both $\mathbf{a}$ and $\mathbf{b}$ represent the linear energy-momentum dispersion of the Dirac cones. The green circles in $\mathbf{b}$ represent the double Dirac cone contours near each $\bar{X}$ point on the surface of $\mathrm{Pb}_{0.6} \mathrm{Sn}_{0.4} \mathrm{Te}$.

found to locate in vicinity of the $\bar{X}$ points. Dispersion measurements (for example, Cut 2 in Fig. 3) reveal a nearly linear dispersion. Spin polarization measurements show that these surface states are spin-polarized and their spin polarization direction is locked with their momentum (spin-momentum locking). The overall electronic structure observed for our surface states are in qualitative agreement with the theoretically predicted surface states of the TCI phase in $\mathrm{SnTe}^{15}$. It is important to note that in an alloy, the space group symmetry is only of the averaged type. The spatial mirror symmetry required for the topological protection is only preserved in a globally averaged sense. To this date, it is not even theoretically known whether only averaged mirror symmetry is sufficient for the predicted TCI phase. In the absence of any theoretical work for the alloy, the experimental proof of mirror protection in the alloy $\mathrm{Pb}_{1-x} \mathrm{Sn}_{x} \mathrm{Te}(\mathrm{Se})$ system perhaps requires the observation of strictly gapless surface states. The current ARPES works ${ }^{29,30}$ have not demonstrated resolution better than $5 \mathrm{meV}$, and thus fitting of the energy-momentum distribution curves lacks the resolving power to exclude gap values $<5 \mathrm{meV}$ (see Supplementary Fig. S10 and Supplementary Discussion). On the other hand, a proof of strictly gapless surface states in fact requires a surface- and spin-sensitive experimental setup with energy resolution better than $1 \mathrm{meV}$, which can even exclude possible small gap values of $\leq 1 \mathrm{meV}$ at the Dirac point. Even a gap size of even $<1 \mathrm{meV}$ can lead to observable change in spin-transport experiments, thus destroying the delicate TCI phase. Therefore, although the existence of surface states correlated with band inversion transition is established here in our work, their gapped or gaplessness nature requires surface transport experiments on samples with improved quality, which is currently not possible ${ }^{29}$.
Our observations of spin-momentum locked surface states in the inverted composition and their absence in the non-inverted composition suggest that $\mathrm{Pb}_{1-x} \mathrm{Sn}_{x} \mathrm{Te}$ system is a tunable spinorbit insulator analogous to the $\operatorname{BiTl}\left(\mathrm{S}_{1-\delta} \mathrm{Se}_{\delta}\right)_{2}$ system ${ }^{5}$, which features a topological phase transition across the band inversion transition. However, the key difference is that odd number of band inversions in the $\operatorname{BiTl}\left(\mathrm{S}_{1-\delta} \mathrm{Se}_{\delta}\right)_{2}$ system leads to odd number of surface states, whereas even number of band inversions in $\mathrm{Pb}_{1-x} \mathrm{Sn}_{x} \mathrm{Te}$ system leads to even number of surface states per surface $\mathrm{BZ}$. We further present a comparison of the $\mathrm{Pb}_{0.6} \mathrm{Sn}_{0.4} \mathrm{Te}$ and a single Dirac cone $\mathrm{Z}_{2} \mathrm{TI} \mathrm{GeBi}_{2} \mathrm{Te}_{4}{ }^{31-33}$. As shown in Figs $5 \mathrm{a}-\mathrm{c}$, for $\mathrm{GeBi}_{2} \mathrm{Te}_{4}$, a single surface Dirac cone is observed enclosing the time-reversal invariant (Kramers') momentum $\bar{\Gamma}$ in both ARPES and calculation results, demonstrating its $Z_{2}$ TI state and the time-reversal symmetry protection of its single Dirac cone surface states. On the other hand, for the $\mathrm{Pb}_{0.6} \mathrm{Sn}_{0.4} \mathrm{Te}$ samples (Figs $5 \mathrm{~d}-\mathrm{f}$ ), none of the surface states is observed to enclose any of the time-reversal invariant momentum, suggesting their irrelevance to the time-reversal symmetry-related protection. With future ultra-high-resolution experimental studies to prove the strict gapless nature of the $\mathrm{Pb}_{0.6} \mathrm{Sn}_{0.4} \mathrm{Te}$ surface states and therefore the topological protection by the crystalline mirror symmetries, it is then possible to realize magnetic yet topologically protected surface states in the $\mathrm{Pb}_{1-x} \mathrm{Sn}_{x} \mathrm{Te}$ system due to its irrelevance to the time-reversal symmetry-related protection, which is fundamentally not possible in the $\mathrm{Z}_{2} \mathrm{TI}$ systems. Considering the wide applications of magnetic materials in modern electronics, such topologically protected surface states compatible with magnetism will be of great interest in terms of integrating TI materials into future electronic devices. 
We note that one advantage of the $\mathrm{Pb}_{1-} \mathrm{Sn}_{x} \mathrm{Te}$ system is that it can be easily doped with manganese, thallium, or indium to achieve bulk magnetic or superconducting states ${ }^{34-36}$. The symmetry in the $\mathrm{Pb}_{1-x} \mathrm{Sn}_{x} \mathrm{Te}$ system (its nonmagnetic character) can be broken by magnetic or superconducting doping into the bulk or the surface. In future experiments, it would be interesting to explore the modification of our observed surface states brought out by magnetic and superconducting correlations, to search for exotic magnetic and superconducting orders on the surface. Such magnetic and superconducting orders on the surface states in $\mathrm{Pb}_{1-} \mathrm{Sn}_{x}$ Te can be different from those recently observed in the $Z_{2}$ TIs $^{37,38}$ because of its very distinct topology of surface electronic structure. The novel magnetic and superconducting states to be realized with this novel topology are not strongly related to the question of gapless or gapped nature of the TCI phase. Therefore, our observation of the spin-polarized surface states presented here provides the much desired platform for realizing unusual surface magnetic and superconducting states in future experiments.

\section{Methods}

Electronic structure measurements. Spin-integrated ARPES measurements were performed with incident photon energies of $8-30 \mathrm{eV}$ at beamline $5-4$ at the Stanford Synchrotron Radiation Lightsource (SSRL) in the Stanford Linear Accelerator Center, and with $28-90 \mathrm{eV}$ photon energies at beamlines 4.0.3, 10.0.1 and 12.0.1 at the Advanced Light Source (ALS) in the Lawrence Berkeley National Laboratory (LBNL). Samples were cleaved in situ between 10 and $20 \mathrm{~K}$ at chamber pressure better than $5 \times 10^{-11}$ torr at both the SSRL and the ALS, resulting in shiny surfaces. Energy resolution was better than $15 \mathrm{meV}$ and momentum resolution was better than $1 \%$ of the surface BZ. To cross-check the cleavage surface orientation in ARPES measurements, the Laue back-reflection X-ray measurements were performed at the ALS using a Realtime Laue delay-line detector from the Multiwire Laboratories (Ithaca, NY), and diffraction spot indexing was performed using the MWL NorthStar orientation package. The X-ray Laue measurements along with the analysis results are presented in the Supplementary Fig. S11.

SD measurements. SR ARPES measurements were performed on the SIS beamline at the Swiss Light Source using the COPHEE spectrometer with two $40 \mathrm{kV}$ classical Mott detectors and a photon energy of $24 \mathrm{eV}$, which systematically measures all three components of the spin of the electron $\left(P_{\mathrm{x}}, P_{\mathrm{y}}\right.$ and $\left.P_{\mathrm{z}}\right)$ as a function of its energy and momentum ${ }^{25,26}$. Energy resolution was bettern than $60 \mathrm{meV}$ and momentum resolution was better than $3 \%$ of the surface BZ for the spin-resolved measurements. Samples were cleaved in situ at $20 \mathrm{~K}$ at a chamber pressure $<2 \times 10^{-10}$ torr. Typical electron counts on the detector reach $5 \times 10^{5}$, which places an error bar of approximately \pm 0.01 for each point on our measured polarization curves.

Sample growth. Single crystals of $\mathrm{Pb}_{1-x} \mathrm{Sn}_{x} \mathrm{Te}$ were grown by the standard growth method (described in ref. 39 for the end compound $\mathrm{PbTe}$ ). High purity (99.9999\%) elements of lead, tin and tellurium were mixed in the right ratio. Lead was etched by $\mathrm{CH}_{3} \mathrm{COOH}+\mathrm{H}_{2} \mathrm{O}_{2}$ (4:1) to remove the surface oxide layer before mixing. The mixture was heated in a clean evacuated quartz tube to $924^{\circ} \mathrm{C}$, where it was held for two days. Afterwards, it was cooled slowly, at a rate of $1.5^{\circ} \mathrm{Ch}^{-1}$ in the vicinity of the melting point, from the high temperature zone towards room temperature. The nominal concentrations were estimated by the $\mathrm{Pb} / \mathrm{Sn}$ mixture weight ratio before the growth, in which the two representative compositions presented in the paper were estimated to be $x_{\text {nom }}=0.20$ and $x_{\text {nom }}=0.36$. The concentration values were re-examined on the single crystal samples after growth using high-resolution X-ray diffraction measurements by tracking the change of the diffraction angle (the $2 \theta$ angle) of the sharpest Bragg peak, from which we obtained $x=0.22$ and $x=0.39$ (see Supplementary Fig. S12 for diffraction data). In the paper we note the chemical compositions as $\mathrm{Pb}_{0.8} \mathrm{Sn}_{0.2} \mathrm{Te}$ and $\mathrm{Pb}_{0.6} \mathrm{Sn}_{0.4} \mathrm{Te}$.

First-principles calculation methods. We perform first-principles calculations to extract both the electronic structure and the spin texture of the SnTe surface states. Our first-principles calculations are within the framework of the density functional theory using full-potential projected augmented wave method ${ }^{40}$, as implemented in the VASP package ${ }^{41}$. The generalized gradient approximation ${ }^{42}$ is used to model exchange-correlation effects. The spin-orbital coupling is included in the selfconsistent cycles. The surfaces are modelled by periodically repeated slabs of 33-atomic-layer thickness, separated by 13 - $\AA$ wide vacuum regions, using a $12 \times 12 \times 1$ Monkhorst-Pack $k$ point mesh over the BZ with $208 \mathrm{eV}$ cutoff energy. The room temperature crystal structures of SnTe in ideal sodium chloride are used to construct the slab to have the required crystal structure and mirror symmetries required for the predicted TCI phase ${ }^{15}$ (the rhombohedral distortion is found to only occur in the Sn-rich compositions $(x>0.5)$ and only at low temperature $\left.{ }^{16}\right)$ The experimental lattice constants of SnTe with the value of $6.327 \AA$ are used ${ }^{43}$. The experimental lattice constant of PbTe with the value of $6.46 \AA^{43}$ in ideal sodium chloride lattice are used in the PbTe band structure calculation.

\section{References}

1. Hasan, M. Z. \& Moore, J. E. Three-dimensional topological insulators. Ann. Rev. Cond. Mat. Phys. 2, 55-78 (2011).

2. Hasan, M. Z. \& Kane, C. L. Topological insulators. Rev. Mod. Phys. 82, 3045-3067 (2010).

3. Qi, X.-L. \& Zhang, S.-C. Topological insulators and superconductors. Rev. Mod. Phys. 83, 1057-1110 (2011).

4. Xia, Y. et al. Observation of a large-gap topological-insulator class with a single Dirac cone on the surface. Nat. Phys. 5, 398-402 (2009).

5. Xu, S.-Y. et al. Topological phase transition and texture inversion in a tunable topological insulator. Science 332, 560-564 (2011).

6. Zogg, H., Fach, A., Masek, J. \& Blunier, S. Photovoltaic lead-chalcogenide on silicon infrared sensor arrays. Opt. Eng. 33, 1440-1449 (1994).

7. Zhu, P. W. et al. Thermoelectric properties of PbTe prepared at high pressure and high temperature. J. Phys. Condens. Matter 14, 11185-11188 (2002).

8. Dimmock, J. O. \& Wright, G. B. Band edge structure of PbS, PbSe, and PbTe. Phys. Rev. 135, 821-830 (1964)

9. Dimmock, J. O., Melngailis, I. \& Strauss, A. J. Band structure and laser action in $\mathrm{Pb}_{x} \mathrm{Sn}_{1-x}$ Te. Phys. Rev. Lett. 16, 1193-1196 (1966).

10. Gao, X. \& Daw, M. S. Investigation of band inversion in (Pb,Sn)Te alloys using ab initio calculations. Phy. Rev. B 77, 033103 (2008).

11. Pankratov, O. A., Pakhomov, S. V. \& Volkov, B. A. Supersymmetry in heterojunctions: band-inverting contact on the basis of $\mathrm{Pb}_{1-{ }_{x}} \mathrm{Sn}_{x} \mathrm{Te}$ and $\mathrm{Hg}_{1-x} \mathrm{Cd}_{x} \mathrm{Te}$. Solid State Commun. 61, 93-96 (1987).

12. Volkov, B. A. \& Pankratov, O. A. Two-dimensional massless electrons in an inverted contact. JETP Lett. 42, 178-181 (1985).

13. Fradkin, E., Dagotto, E. \& Boyanovsky, D. Physical realization of the parity anomaly in condensed matter physics. Phys. Rev. Lett. 57, 2967-2970 (1986).

14. Fu, L. Topological crystalline insulators. Phys. Rev. Lett. 106, 106802 (2011).

15. Hsieh, H. et al. Topological crystalline insulators in the SnTe material class. Nat. Comm. 3, 982 (2012).

16. Iizumi, M. et al. Phase transition in SnTe with low carrier concentration. J. Phys. Soc. Jpn. 38, 443-449 (1975).

17. Burke, Jr J. R., Allgaier, R. S., Houston, Jr B. B., Babiskin, J. \& Siebenmann, P. G. Shubnikov-de Haas effect in SnTe. Phys. Rev. Lett. 14, 360-361 (1965).

18. Littlewood, P. B. et al. Band structure of SnTe studied by photoemission spectroscopy. Phys. Rev. Lett. 105, 086404 (2010).

19. Takafuji, Y. \& Narita, S. Shubnikov-de Haas measurements in N-Type $\mathrm{Pb}_{1-x} \mathrm{Sn}_{x}$ Te. Jpn. J. Appl. Phys. 21, 1315-1322 (1982).

20. Yannopapas, V. Gapless surface states in a lattice of coupled cavities: a photonic analog of topological crystalline insulators. Phys. Rev. B 84, 195126 (2011).

21. Hao, N., Zhang, P. \& Wang, Y. Topological phases and fractional excitations of the exciton condensate in a special class of bilayer systems. Phys. Rev. B 84, 155447 (2011).

22. Vildanov, N. M. Effective field theory description of topological crystalline insulators. Preprint at http://arXiv.org/abs/1205.3560 (2012).

23. Hsieh, D. et al. A topological Dirac insulator in a quantum spin Hall phase. Nature 452, 970-974 (2008).

24. Fu, L. Hexagonal warping effects in the surface states of the topological insulator $\mathrm{Bi}_{2} \mathrm{Te}_{3}$. Phys. Rev. Lett. 103, 266801 (2009).

25. Hoesch, M. et al. Spin-polarized Fermi surface mapping. J. Electron Spectrosc. Relat. Phenom. 124, 263-279 (2002).

26. Dil, J. H. et al. Spin and angle resolved photoemission on non-magnetic lowdimensional systems. J. Phys. Condens. Matter 21, 403001 (2009).

27. Teo, J. C. Y., Fu, L. \& Kane, C. L. Surface states and topological invariants in three-dimensional topological insulators: application to $\mathrm{Bi}_{1-x} \mathrm{Sb}_{x}$. Phys. Rev. B 78, 045426 (2008).

28. Hsieh, D. et al. Observation of unconventional quantum spin textures in topological insulators. Science 323, 919-922 (2009).

29. Dziawa, P. et al. Topological crystalline insulator states in $\mathrm{Pb}_{1-{ }_{x}} \mathrm{Sn}_{x} \mathrm{Se}$. Nat Mater. doi:10.1038/nmat3449 (2012).

30. Xu, S.-Y. et al. Observation of topological crystalline insulator phase in the lead tin chalcogenide $\mathrm{Pb}_{1-\mathrm{x}} \mathrm{SnxTe}$ material class. Preprint at http://arXiv.org/abs/ 1206.2088 (2012).

31. Xu, S.-Y. et al. Discovery of several large families of topological insulator classes with backscattering-suppressed spin-polarized single-Dirac-cone on the surface. Preprint at http://arXiv.org/abs/1007.5111 (2010).

32. Neupane, M. et al. Topological surface states and Dirac point tuning in ternary topological insulators. Phys. Rev. B 85, 235406 (2012).

33. Okamoto, K. et al. Observation of a highly spin polarized topological surface state in $\mathrm{GeBi}_{2} \mathrm{Te}_{4}$. Preprint at http://arXiv.org/abs/1207.2088 (2012). 
34. Story, T. et al. Carrier-concentration-induced ferromagnetism in PbSnMnTe. Phys. Rev. Lett. 56, 777-779 (1986).

35. Nakayama, K., Sato, T., Takahashi, T. \& Murakami, H. Doping induced evolution of Fermi surface in low carrier superconductor Tl-doped PbTe. Phys. Rev. Lett. 100, 227004 (2008).

36. Sasaki, S. et al. Odd-parity pairing and topological superconductivity in a strongly spin-orbit coupled semiconductor. Preprint at http://arXiv.org/abs/ 1208.0059 (2012).

37. Wray, L. A. et al. Observation of topological order in a superconducting doped topological insulator. Nat. Phys. 6, 855-859 (2010).

38. Xu, S.-Y. et al. Hedgehog spin texture and Berry's phase tuning in a magnetic topological insulator. Nat. Phys. 8, 616-622 (2012).

39. Nugraha, K., Suto, O., Itoh, J. \& Nishizawa, Y. Yokota, growth and electrical properties of PbTe bulk crystals grown by the Bridgman method under controlled tellurium or lead vapor pressure. J. Cryst. Gr. 165, 402-407 (1996).

40. Kresse, G. \& Joubert, D. From ultrasoft pseudopotentials to the projector augmented-wave method. Phys. Rev. B 59, 1758-1775 (1999).

41. Perdew, J. P., Burke, K. \& Ernzerhof, M. Generalized gradient approximation made simple. Phys. Rev. Lett. 77, 3865-3868 (1996).

42. Kresse, G. \& Hafner, J. Ab initio molecular dynamics for open-shell transition metals. Phys. Rev. B 48, 13115-13118 (1993).

43. Bis, R. F. \& Dixon, J. R. Applicability of Vagard's law to the $\mathrm{Pb}_{x} \mathrm{Sn}_{1-x}$ Te alloy system. J. Appl. Phys. 40, 1918-1921 (1969).

\section{Acknowledgements}

We thank L. Fu and A. Vishwanath for theoretical discussions on TCI. The work at Princeton and Princeton-led synchrotron $\mathrm{x}$-ray-based measurements and the related theory at Northeastern University are supported by the Office of Basic Energy Sciences, US Department of Energy (grants DE-FG-02-05ER46200, AC03-76SF00098 and DE-FG02-07ER46352). M.Z.H. acknowledges visiting-scientist support from the Lawrence Berkeley National Laboratory and additional support from the A. P. Sloan
Foundation. The spin-resolved and spin-integrated photoemission measurements using synchrotron X-ray facilities are supported by the Advanced Light Source, the Stanford Synchrotron Radiation Lightsource, and the Swiss Light Source, by the Basic Energy Sciences of the US Department of Energy and the Swiss National Science Foundation. Theoretical computations benefited from the allocation of supercomputer time at NERSC and Northeastern University's Advanced Scientific Computation Center. Sample growth and characterization are partially supported by DMR-0819860, DMR-1006492 and by the National Science Council (NSC)-Taiwan under project number NSC100-2119-M002-021. We gratefully acknowledge N. Lavdovsky for assistance with sample preparation. We also thank M. Hashimoto and S.-K. Mo for beamline assistance at the Stanford Synchrotron Radiation Lightsource and the Advanced Light Source in Berkeley.

\section{Author contributions}

S.-Y.X. and C.L. performed the experiments with assistance from N.A., M.N., D.Q., I.B., L.A.W. and M.Z.H. A.M., E.M., Q.G., R.S., F.C.C. and R.J.C. provided samples; G.L., B.S. J.H.D. and J.D.D. provided beamline assistance; Y.J.W., H.L. and A.B. carried out the theoretical calculations; M.Z.H. was responsible for the conception and the overall direction, planning and integration among different research units.

\section{Additional information}

Supplementary Information accompanies this paper at http://www.nature.com/ naturecommunications

Competing financial interests: The authors declare no competing financial interests.

Reprints and permission information is available online at http://npg.nature.com/ reprintsandpermissions/

How to cite this article: Xu, S.-Y. et al. Observation of a TCI phase and topological phase transition in $\mathrm{Pb}_{1-x} \mathrm{Sn}_{x}$ Te. Nat. Commun. 3:1192 doi: 10.1038/ncomms2191 (2012). 


\section{Corrigendum: Observation of a topological crystalline insulator phase and topological phase transition in $\mathrm{Pb}_{1-x} \mathrm{Sn}_{x} \mathrm{Te}$}

Su-Yang Xu, Chang Liu, N. Alidoust, M. Neupane, D. Qian, I. Belopolski, J.D. Denlinger, Y.J. Wang, H. Lin, L.A. Wray, G. Landolt, B. Slomski, J.H. Dil, A. Marcinkova, E. Morosan, Q. Gibson, R. Sankar, F.C. Chou, R.J. Cava, A. Bansil \& M.Z. Hasan

Nature Communications 3:1192 doi: 10.1038/ncomms2191 (2012); Published 13 Nov 2012; Updated 4 Aug 2016

In Fig. 2a and $\mathrm{d}$ of this Article, a uniform background subtraction was applied to the ARPES low-energy electronic structure measurements. In Fig. 2d, this background subtraction unintentionally was only applied to the central part of the figure. This affected the noise in the background but not the meaningful signal data features discussed in the paper, and therefore does not affect any conclusion of the paper. To avoid potential confusion, in the updated version of Fig. $2 \mathrm{a}$ and d, raw data without any background subtraction are presented. The corrected version of Fig. 2 appears below as Fig. 1. The figure legend is correct. 

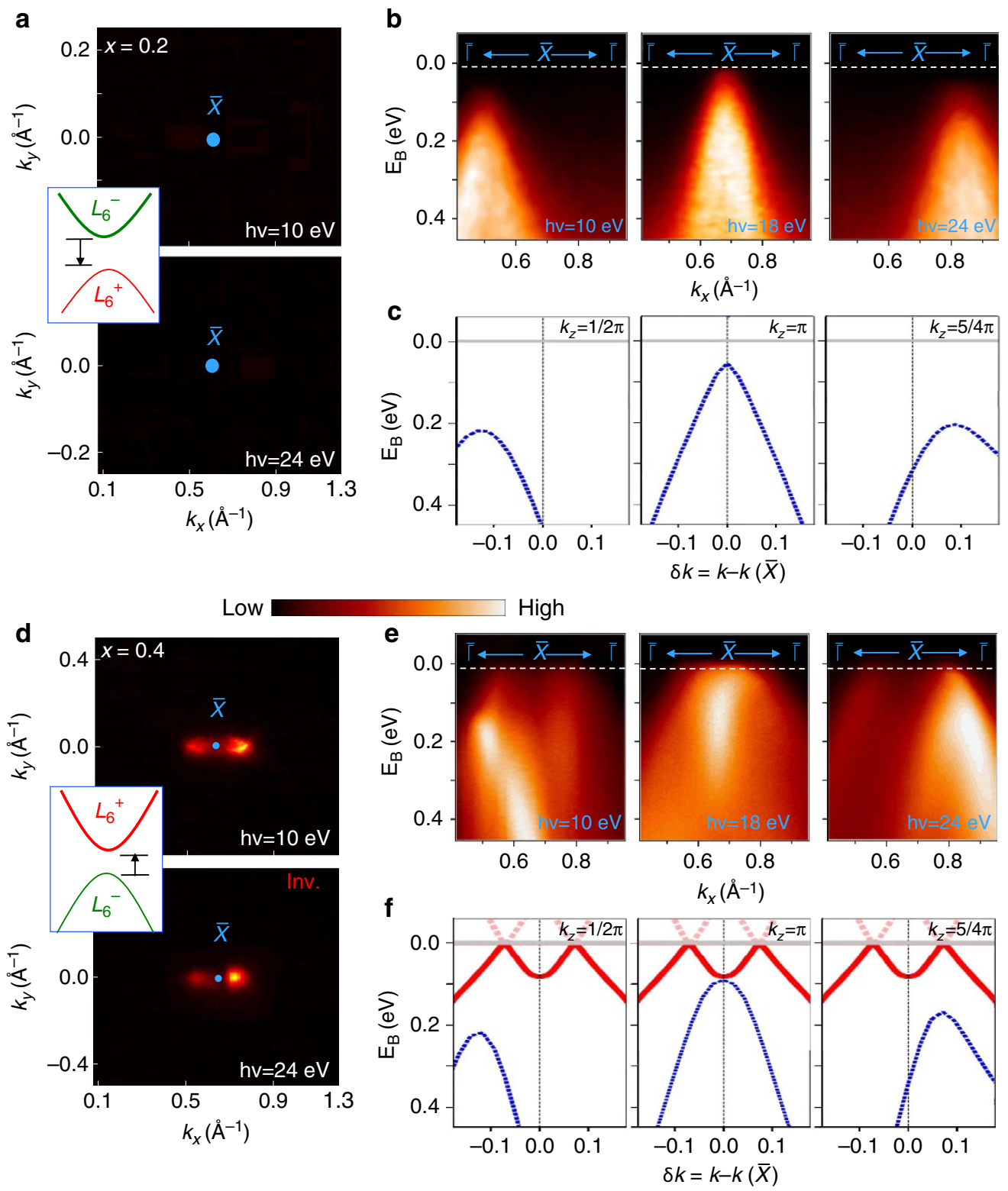

Figure 1 